\title{
Fabrication and Characterization of Collagen-Immobilized Porous PHBV/HA Nanocomposite Scaffolds for Bone Tissue Engineering
}

\author{
Jin-Young Baek, Zhi-Cai Xing, Giseop Kwak, Keun-Byoung Yoon, Soo-Young Park, \\ Lee Soon Park, and Inn-Kyu Kang
}

Department of Polymer Science and Engineering, Kyungpook National University, Daegu 702-701, Republic of Korea

Correspondence should be addressed to Inn-Kyu Kang, ikkang@knu.ac.kr

Received 6 December 2011; Revised 3 February 2012; Accepted 6 February 2012

Academic Editor: Zhongkui Hong

Copyright ( $) 2012$ Jin-Young Baek et al. This is an open access article distributed under the Creative Commons Attribution License, which permits unrestricted use, distribution, and reproduction in any medium, provided the original work is properly cited.

\begin{abstract}
The porous composite scaffolds (PHBV/HA) consisting of poly (3-hydroxybutyrate-co-3-hydroxyvalerate) (PHBV) and hydroxyapatite (HA) were fabricated using a hot-press machine and salt-leaching. Collagen (type I) was then immobilized on the surface of the porous PHBV/HA composite scaffolds to improve tissue compatibility. The structure and morphology of the collagen-immobilized composite scaffolds (PHBV/HA/Col) were investigated using a scanning electron microscope (SEM), Fourier transform infrared (FTIR), and electron spectroscopy for chemical analysis (ESCA). The potential of the porous $\mathrm{PHBV/HA/Col} \mathrm{composite} \mathrm{scaffolds} \mathrm{for} \mathrm{use} \mathrm{as} \mathrm{a} \mathrm{bone} \mathrm{scaffold} \mathrm{was} \mathrm{assessed} \mathrm{by} \mathrm{an} \mathrm{experiment} \mathrm{with} \mathrm{osteoblast} \mathrm{cells} \mathrm{(MC3T3-E1)} \mathrm{in}$ terms of cell adhesion, proliferation, and differentiation. The results showed that the PHBV/HA/Col composite scaffolds possess better cell adhesion and significantly higher proliferation and differentiation than the PHBV/HA composite scaffolds and the PHBV scaffolds. These results suggest that the PHBV/HA/Col composite scaffolds have a high potential for use in the field of bone regeneration and tissue engineering.
\end{abstract}

\section{Introduction}

Polyhydroxyalkanoates (PHAs) are natural biodegradable thermoplastics that are accumulated by a wide variety of microorganisms as a unique intracellular storage of carbon, among which poly (3-hydroxybutyric acid) (PHB) and poly (3-hydroxybutyric acid-co-3-hydroxyvaleric acid) (PHBV) are two main products [1]. Their inherent biodegradability, nontoxicity, and biocompatibility make them suitable for biomaterials in tissue engineering [2-4]. Over the past three decades, hydroxyapatite (HA), which is similar to the mineral component of natural bone, has been extensively studied and now used for bone tissue repair [5]. HA is considered to be osteoconductive, that is, capable of promoting bone formation, due to its capability to bind and concentrate bone morphogenetic proteins (BMPs) in vivo.

Efforts have thus been made to form nonporous $\mathrm{PHB} /$ $\mathrm{HA}$ and $\mathrm{PHBV} / \mathrm{HA}$ composites for bone tissue repair by utilizing the osteoconductive property of HA [6-8]. Sultana and wang [9] prepared PHBV/HA scaffold using an emulsion freezing/freeze-drying technique. They found that the scaffolds were highly porous and had interconnected porous structures, and the incorporation of HA nanoparticles enhanced compressive mechanical properties of the scaffolds. Jack et al. [10] fabricated the PHBV/HA composite scaffolds with high porosity and controlled pore architectures. Their results showed that the incorporation of HA nanoparticles increased the stiffness and strength and improved the in vitro bioactivity of the scaffolds. For bone tissue engineering, biodegradable composite scaffolds containing HA appear to hold great promises.

For a given environment, the cellular interaction with a polymer is strongly dependent on the surface characteristics (i.e., topography and chemistry) of the polymer [11-14]. Polymer surface chemistry has been shown to influence the initial cell adhesion through the adsorption of proteins 


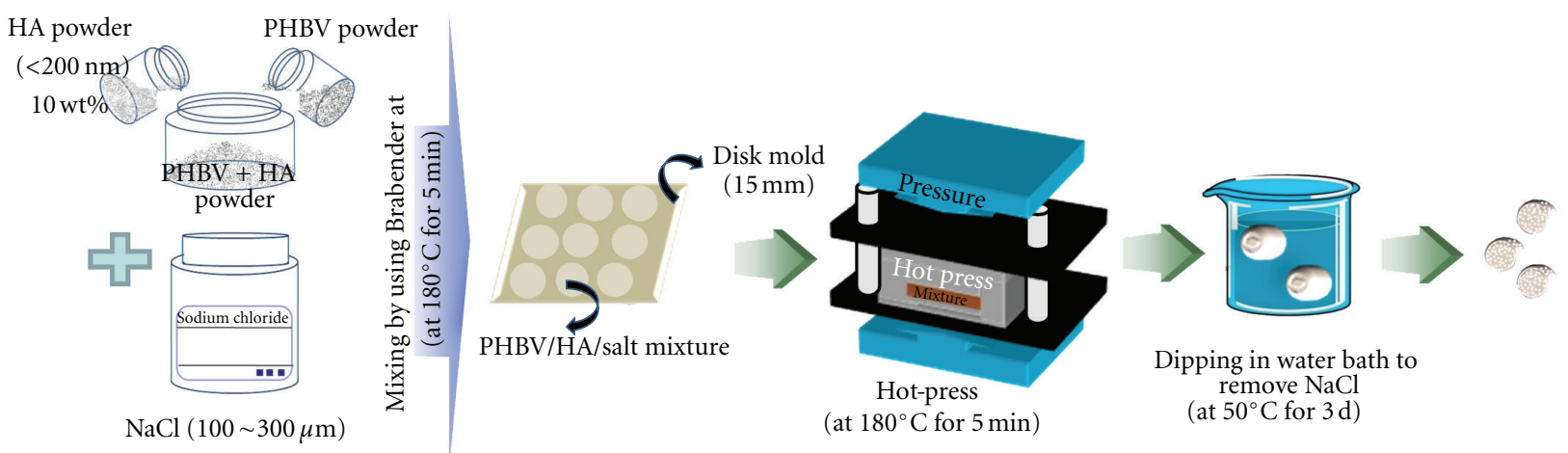

Scheme 1: Preparation of porous PHBV/HA scaffolds.
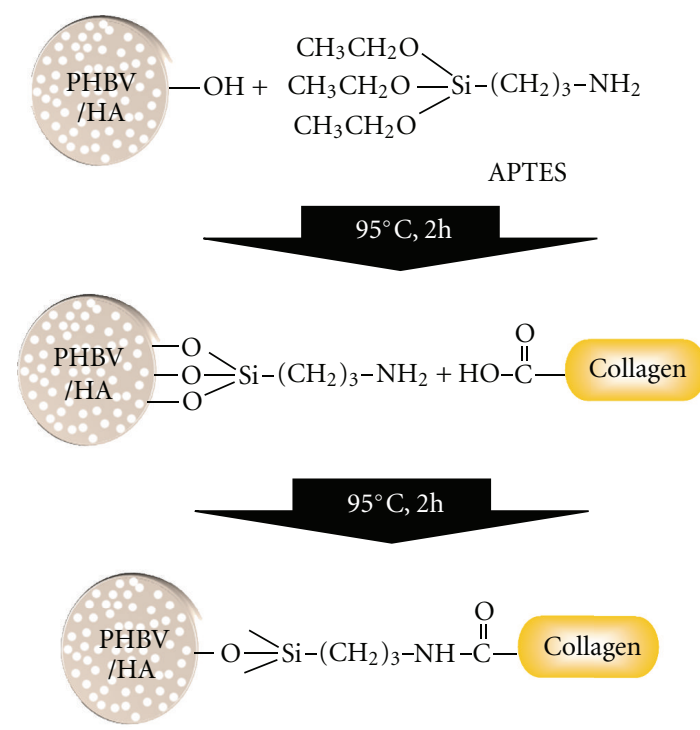

Scheme 2: Collagen immobilization of porous PHBV/HA scaffolds.

derived from the serum used in the culture medium. The cell adhesion is believed to involve the adhesion receptors on the cell membrane and extracellular matrix (ECM) proteins adsorbed to the polymer surface. To mimic the natural environment of connective tissue, the polymeric surfaces have been modified by coating or grafting ECM proteins (fibronectin, vitronectin, and collagen) that have a cellbinding domain containing the RGD sequence [15-19].

Collagen is one of the most abundant structural proteins found in animal connective tissues and is an important protein for anchoring cells such as fibroblasts or epithelium [20]. Depending on the final application (cell carrier/scaffold), the natural protein is either grafted (chemically immobilized) or dip coated (physically immobilized) on the polymer surface $[14,21]$. Tesema et al. concluded that the collagenimmobilized PHBV membrane provided a more favorable matrix for cell proliferation than the collagen dip-coated PHBV porous membranes or unmodified PHBV porous membranes [22]. Our previous study showed collagenimmobilized $\mathrm{HA} / \mathrm{TiO}_{2}$ composite nanofiber mats possess better cell adhesion and significantly higher proliferation and differentiation than the untreated $\mathrm{HA} / \mathrm{TiO}_{2}$ composite nanofiber mats [23]. However, immobilization of collagen on the porous PHBV/HA scaffolds has not been reported. The combination of PHBV, HA, and collagen in a porous scaffold should give the combined benefits of properties which are not achievable by individual components. Based on these beneficial properties, three-dimensional porous scaffolds could be applied in dental and medical fields.

In this study, PHBV/HA composite scaffolds were obtained by the salt-leaching method. Collagen (type 1) was then immobilized on the surface of the porous $\mathrm{PHBV} / \mathrm{HA}$ scaffolds (PHBV/HA/Col) to enhance the tissue compatibility. The morphology of the porous $\mathrm{PHBV} / \mathrm{HA} / \mathrm{Col}$ scaffolds was investigated and compared with that of the unmodified PHBV/HA, and PHBV scaffolds. Cell behaviors on $\mathrm{PHBV} / \mathrm{HA} / \mathrm{Col}, \mathrm{PHBV} / \mathrm{HA}$, and PHBV scaffolds were compared, in terms of cell adhesion, proliferation, and differentiation to study the potential for use in the field of bone regeneration. 


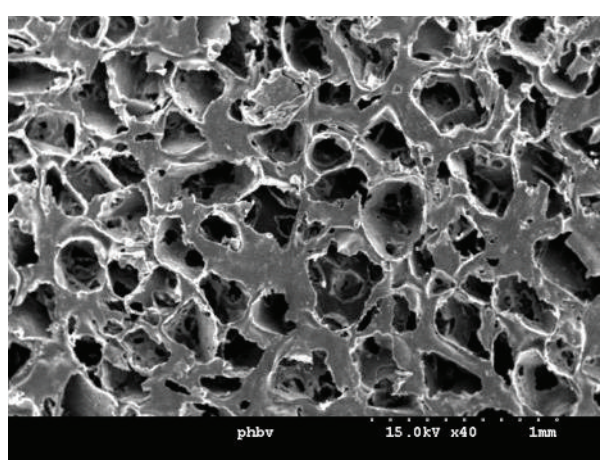

(a)

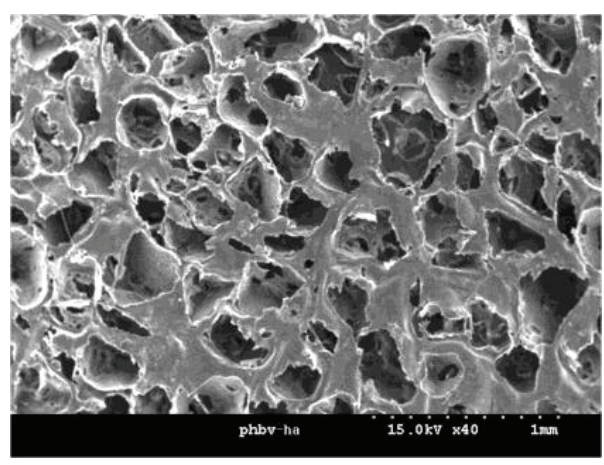

(b)

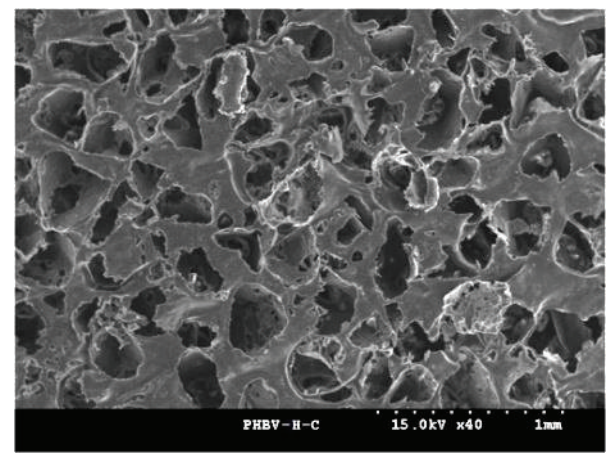

(c)

Figure 1: FE-SEM images of porous PHBV (a), PHBV/HA (b), and PHBV/HA/Col (c) scaffolds.

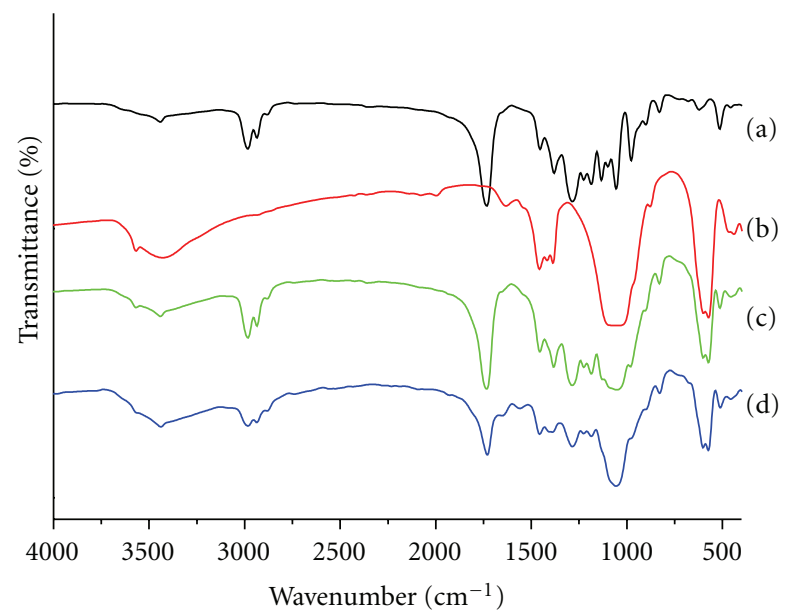

Figure 2: FT-IR spectra of the PHBV (a), HA (b), PHBV/HA (c), and PHBV/HA/Col (d) scaffolds.

\section{Experimental}

2.1. Scaffold Preparation. Porous poly(3-hydroxybutyric acidco-3-hydroxyvaleric acid) (PHBV, hydroxyvaleric acid content: $5 \mathrm{wt} \%$; Aldrich Chemical Co. USA)/HA scaffolds were prepared by mixing the PHBV/HA powder $(\mathrm{w} / \mathrm{w}=$ $9: 1)$ with sodium chloride (PHBV/HA $: \mathrm{NaCl}=1: 17)$, followed by salt-leaching techniques (Scheme 1). The sodium chloride particles of size $100 \sim 300 \mu \mathrm{m}(75 \mathrm{~g})$ were mixed with PHBV/HA powder ( $4.0 \mathrm{~g}$ ) using a Brabender machine
(Plasti-corder Co. Korea) for 5 minutes at $180^{\circ} \mathrm{C}$. The resulting mixture was cast in a mold (Diameter: $15 \mathrm{~mm}$, thickness: $2 \mathrm{~mm}$ ) for 5 minutes at $180^{\circ} \mathrm{C}$. The resulting $\mathrm{PHBV} / \mathrm{HA} / \mathrm{NaCl}$ composite scaffolds were immersed in a shaker containing $100 \mathrm{~mL}$ of distilled water at $50^{\circ} \mathrm{C}$ for 3 days (the water was changed every 12 hours) to leach out the sodium chloride. The salt-free PHBV/HA scaffolds were air and vacuum dried for 24 hours and stored in a dessicator for further use. The drying process was continued until the constant weight of the scaffolds could be obtained. 


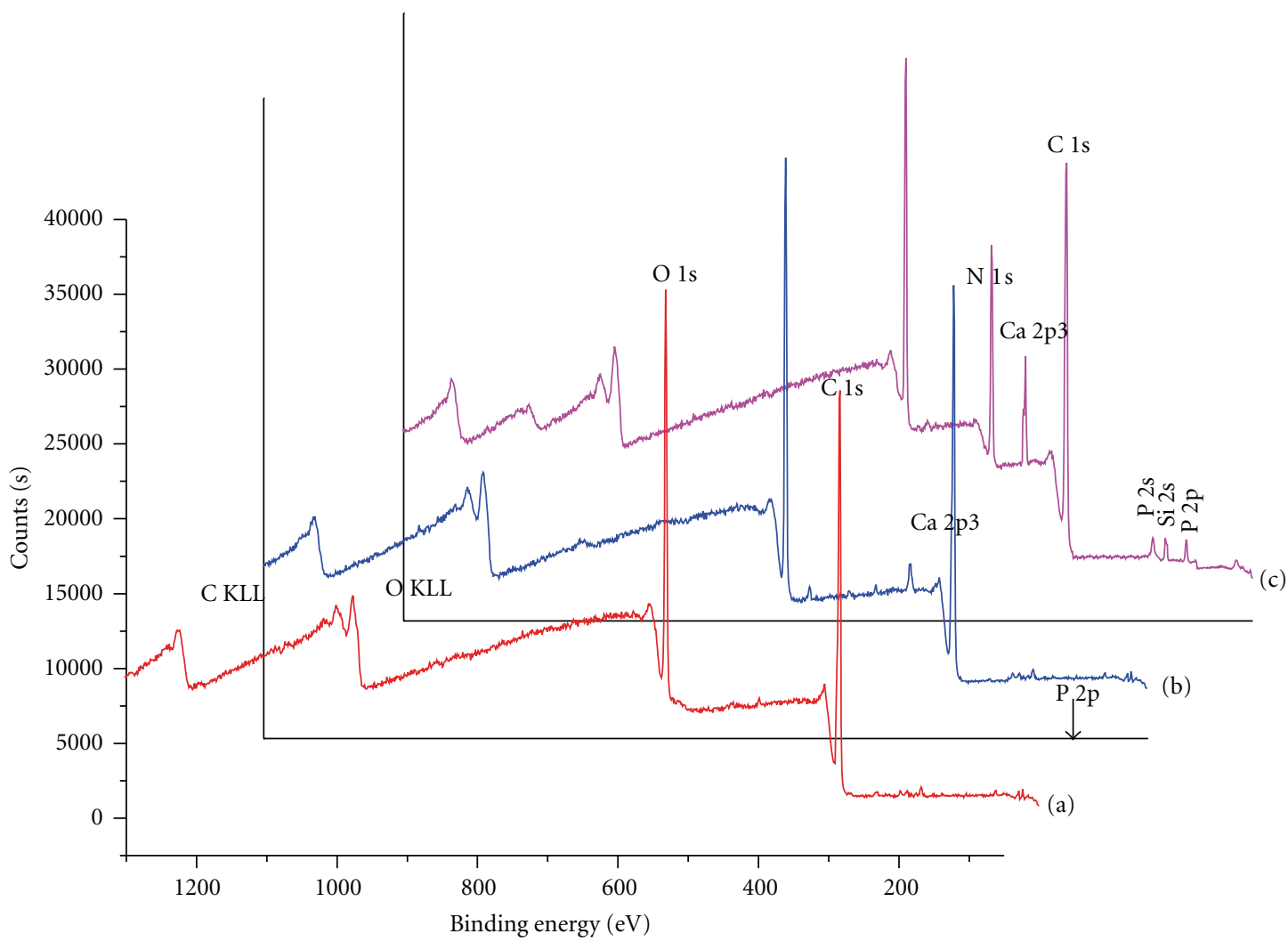

FIgURe 3: ESCA scan spectra and chemical composition of a PHBV (a), PHBV/HA (b), and PHBV/HA/Col (c) scaffolds.

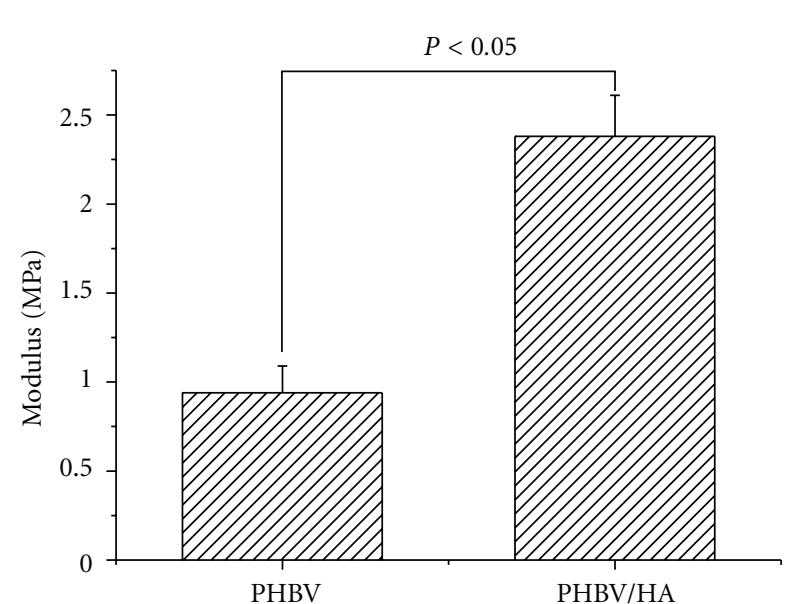

(a)

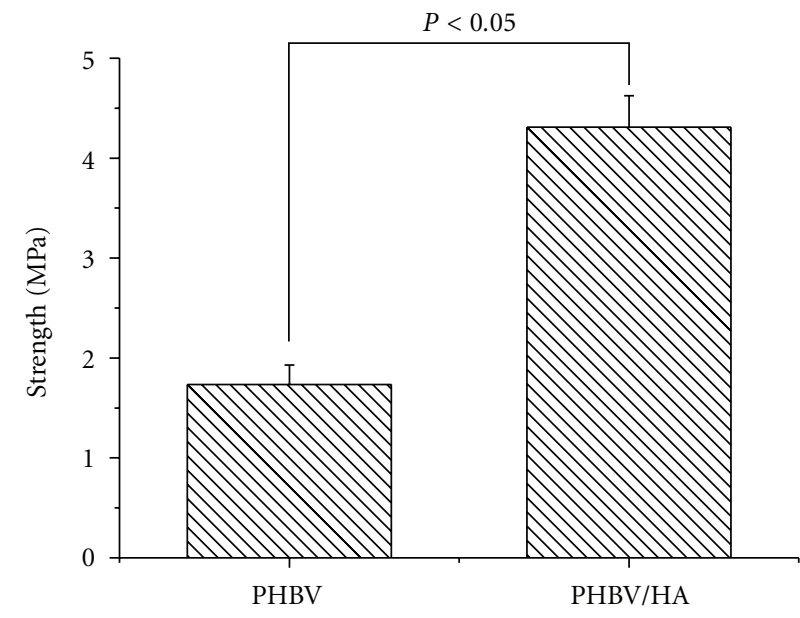

(b)

FIgure 4: Tensile properties of the PHBV and PHBV/HA scaffolds.

2.2. Immobilization of Collagen on $P H B V / H A$ Composite Scaffolds. The immobilization of collagen (type 1, Bioland Technology Ltd, Korea) on the surface of PHBV/HA scaffolds carried out in two steps (Scheme 2). First, an amine group $\left(-\mathrm{NH}_{2}\right)$ was introduced to the surface of the PHBV/HA composite scaffolds by a silanization reaction using 3aminopropyltriethoxysilane (APTES) [24]. Then, collagen was immobilized on the surface of the APTES-modified
PHBV/HA composite scaffolds using water soluble carbodiimide (WSC) [24]. The PHBV/HA/Col composite scaffolds were then washed in distilled water and dried.

2.3. Surface Characterization. The morphology of the composite scaffolds was examined using a field emission scanning electron microscope (FE-SEM S4300, Hitachi, Tokyo, Japan). 


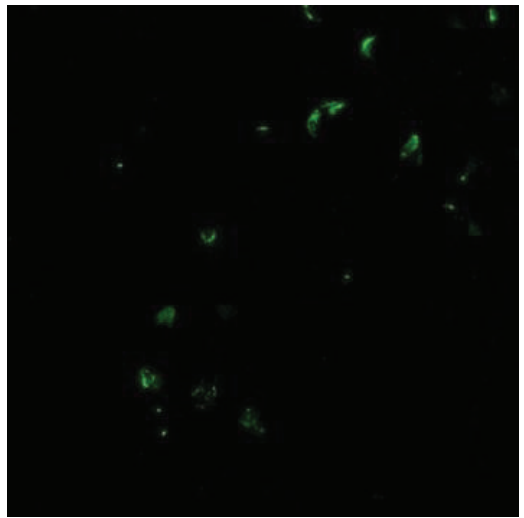

(a)

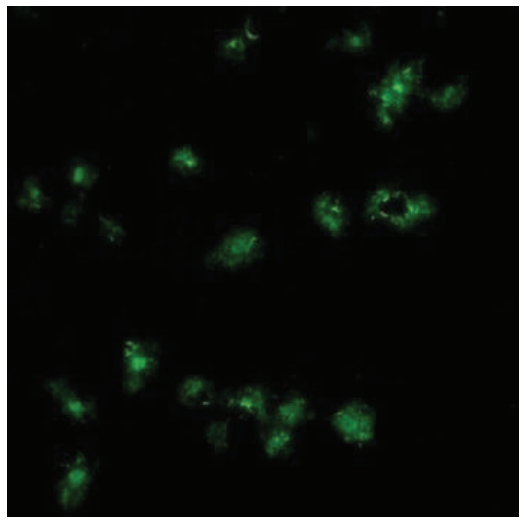

(d)

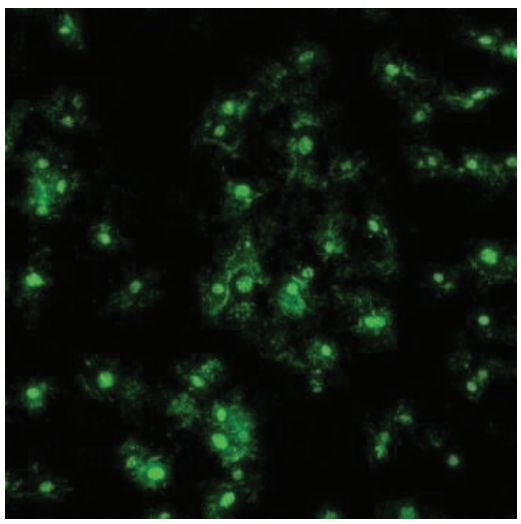

(g)

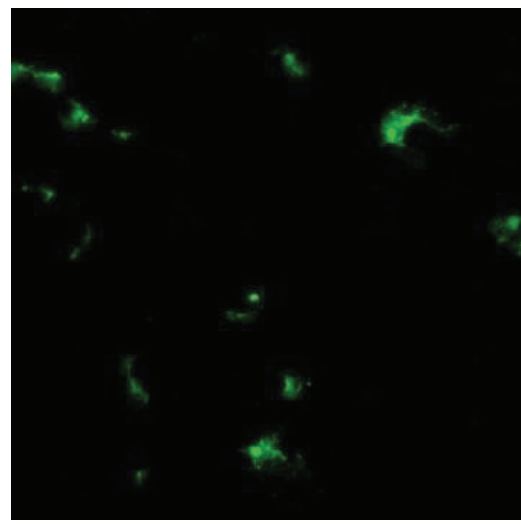

(b)

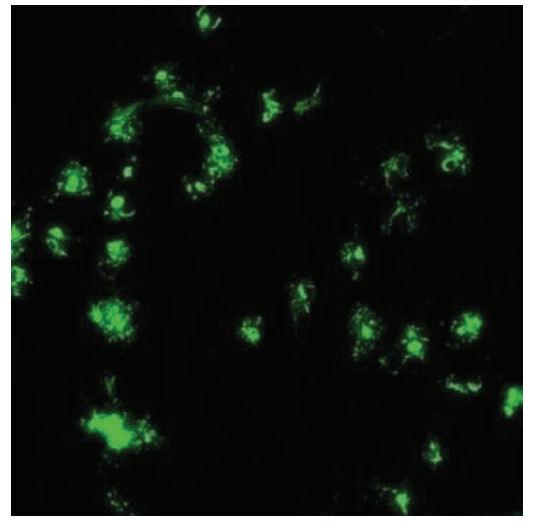

(e)

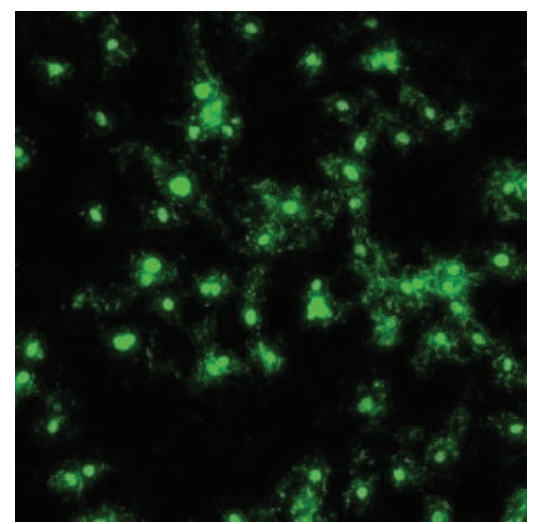

(h)

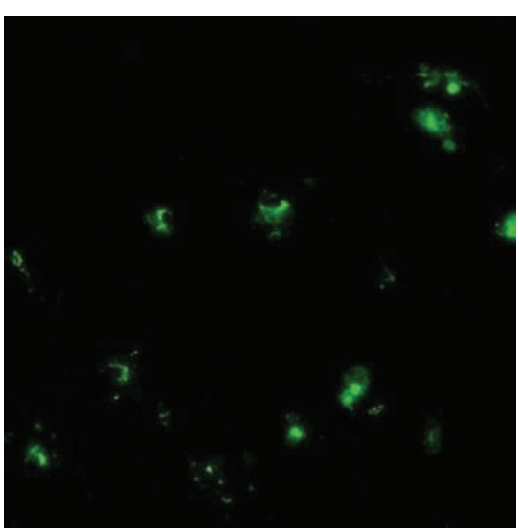

(c)

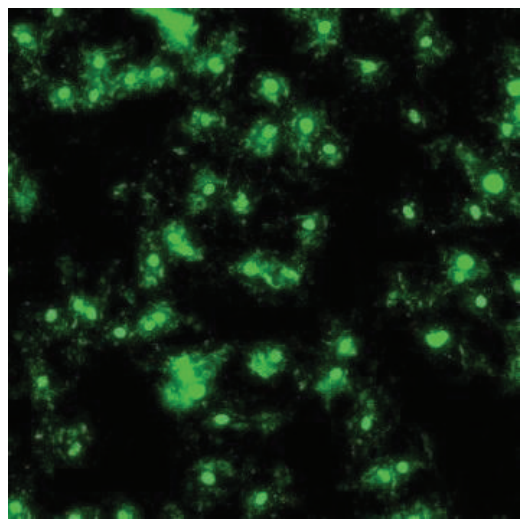

(f)

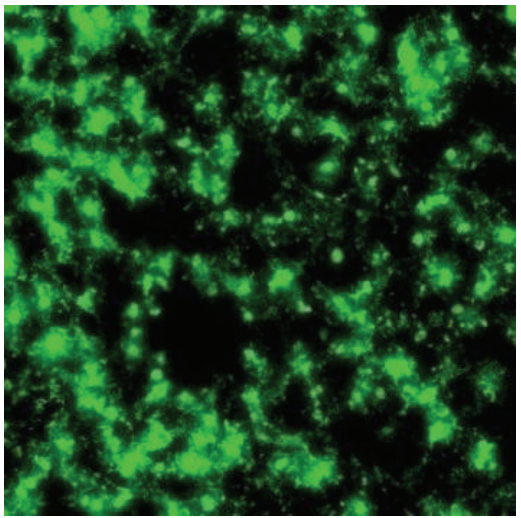

(i)

FIGURE 5: Confocal laser scanning microscope images of calcein-AM dye-stained osteoblasts on PHBV (a, d, g), PHBV/HA (b, e, h) and PHBV/HA/Col (c, f, i) scaffolds for 4 hours (a, b, c,), 1 day (d, e, f), and 2 days (g, h, i) (magnification 100×).

Fourier transform infrared (FTIR, Mattson, Galaxy 7020A) spectroscopy was used to identify the chemical bond and ionic groups in the composite scaffolds. The surface chemical composition was analyzed using electron spectroscopy for chemical analysis (ESCA, ESCA LAB VIG microtech, Mt 500/1, and so forth, East Grinstead, UK), which was equipped with $\mathrm{Mg} \mathrm{K} \alpha$ at $1253.6 \mathrm{eV}$ and a $150 \mathrm{~W}$ power mode at the anode. A survey scan spectrum was taken and the surface elemental compositions relative to the carbon that took into account sensitivity were calculated from the peak heights.

2.4. Mechanical Properties. According to the ASTM Standard D3379-75, tensile test for single specimen was conducted on a Zwick Roell Tensile tester equipped with a $1 \mathrm{kgf}$ load cell. The size of the sample for the strength test was $100 \mathrm{~mm}$ in length, $10 \mathrm{~mm}$ in width, and $2 \mathrm{~mm}$ in thickness. A tensile test was carried out using a universal testing machine (UTM, 
Instron, model 4465$)$ at $25^{\circ} \mathrm{C}$ with an extension speed of $10 \mathrm{~mm} / \mathrm{min}$. The results reported were the averages of 10 specimens for each scaffold.

2.5. Cell Culture. In order to examine the interaction of the composite scaffolds (PHBV, PHBV/HA, and PHBV/HA/Col) with osteoblasts (MC3T3-E1), the circular scaffolds were fitted into a 24-well culture dish using glass ring and immersed in a DMEM medium containing $10 \%$ fetal bovine serum (FBS, Gibco, Invitrogen, USA). Then, $1 \mathrm{~mL}$ of the MC3T3-E1 cell solution $\left(3 \times 10^{4}\right.$ cells $\left./ \mathrm{mL}\right)$ was added to the mats and then incubated in a humidified atmosphere of $5 \%$ $\mathrm{CO}_{2}$ at $37^{\circ} \mathrm{C}$ for 4 hours, 1 day, and 2 days. After incubation, the supernatant was removed and calcein-AM $(1 \mathrm{mM}$ in PBS) solution was added and left standing for 15 minutes. Cells with intact cell membranes are able to use nonspecific cytosolic esterases to convert nonfluorescent calcein-AM into bright green-fluorescent calcein. The fluorescence images were visualized with a confocal laser scanning microscope (CLSM 700, Carl Zeiss, Germany) after washing the scaffolds three times (10 minutes each) with a PBS solution [25]. The morphology of the cells incubated for 5 days was also observed with a confocal laser scanning microscope (CLSM 700, Carl Zeiss, Germany).

2.6. Evaluation of Cell Proliferation. The proliferation of MC3T3-E1 cells was determined using the enzyme-linked immunosorbent assay (ELISA) [26, 27]. The ELISA method is based on the measurement of 5-bromo-2-deoxyuridine (BrdU), which is incorporated during DNA synthesis. The ELISA was done according to the manufacturer's instructions (Roche Molecular Biochemicals, Germany).

2.7. Cytoskeletal Organization. Osteoblast cells were seeded onto the scaffolds $\left(2 \times 10^{4}\right.$ cells $\left./ \mathrm{mL}\right)$ and cultured for 3 days. At each time point, the cells were fixed in $4 \%$ paraformaldehyde in PBS. The samples were washed after fixation with a buffer solution (PBS solution containing $0.05 \%$ Tween-20) and permeabilized with $0.1 \%$ Triton X100 in PBS for 15 minutes at $25^{\circ} \mathrm{C}$. The samples were then incubated for 30 minutes in 1\% BSA/PBS followed by the addition of TRITC-conjugated Phalloidin (Millipore, Cat. no. 90228) for 1 hour. The samples were washed three times (10 minutes each) with buffer solution and incubated with DAPI (Millipore Cat. no. 90229) for 5 minutes. The fluorescence images were visualized with a confocal laser scanning microscope (CLSM 700, Carl Zeiss, Germany) after washing the scaffolds three times (10 minutes each) with buffer solution [28].

2.8. Alkaline Phosphatase (ALP) Activity. The differentiation of osteoblast cells was evaluated by the expression of alkaline phosphatase (ALP) and its activity [29]. After 15 days of culturing the osteoblasts on the $\mathrm{PHBV}, \mathrm{PHBV} / \mathrm{HA}$ and PHBV/HA/Col nanofibrous scaffolds, ALP staining was done by a standard procedure [30] according to the manufacturer's instructions (Alkaline phosphatase, Leukocyte, Procedure no. 86, Sigma-Aldrich, USA). After culturing, the osteoblast cells were washed with a PBS solution and fixed with a citrate-acetone-formaldehyde fixative solution (citrate solution $25 \mathrm{~mL}$, acetone $65 \mathrm{~mL}$, and formaldehyde solution $10 \mathrm{~mL}$ ) at room temperature for 30 seconds. Subsequently, the cell fixed discs were rinsed three times with PBS solution for 45 seconds and stained with alkaline-dye mixture (sodium nitrate $1 \mathrm{~mL}, \mathrm{FBB}$-alkaline solution $1 \mathrm{~mL}$, naphthol AS-BI alkaline solution $1 \mathrm{~mL}$, and deionized water $45 \mathrm{~mL}$ ) at room temperature for 15 minutes. After removing the dye solution, the dyed samples were washed twice with distilled water to completely remove the redundant stains and then dried. The cells stained positively for ALP were observed with an optical microscope (Carl Zeiss, Germany).

2.9. Statistical Analysis. Our results are displayed as the mean \pm standard deviation. The statistical significance of the differences between the scaffolds was determined by a Student's two-tailed $t$ test. Scheffe's method was used for multiple comparison tests at a level of $95 \%$.

\section{Results and Discussion}

3.1. Surface Characterization. The SEM images of the porous $\mathrm{PHBV}$ (a), PHBV/HA (b), and PHBV/HA/Col (c) composite scaffolds are shown in Figure 1. PHBV scaffolds exhibiting interconnected but porous networks with pore sizes ranging from several microns to around $400 \mu \mathrm{m}$ were made (Figure 1(a)). Under the same processing parameters, the resulting PHBV/HA composite scaffolds possessed nearly the same porous morphology (Figure $1(\mathrm{~b})$ ) as the PHBV scaffolds. After collagen immobilization, the pore size of the composite scaffolds decreased slightly and the scaffolds exhibited both open and closed pore morphologies (Figure 1(c)).

The chemical bonding structure of the PHBV, HA, $\mathrm{PHBV} / \mathrm{HA}$, and $\mathrm{PHBV} / \mathrm{HA} / \mathrm{Col}$ scaffolds were examined by FT-IR spectroscopy, as shown in Figure 2. The spectra of the PHBV/HA composite scaffolds (Figure 2(c)) displayed the vibrational bands at $1722 \mathrm{~cm}^{-1}$ based on $\mathrm{C}=$ $\mathrm{O}$ bond of PHBV and $1039 \mathrm{~cm}^{-1}$ based on $\mathrm{PO}_{4}^{3-}$ of the hydroxyapatite, indicating the presence of PHBV and HA. In the PHBV/HA/Col scaffold (Figure 2(d)), characteristic adsorptions were observed at 1640 and $1572 \mathrm{~cm}^{-1}$ based on amide I (-CONH-) and II (-CONH-) bonds of collagen, respectively $[23,31,32]$. This result suggests that collagen was successfully immobilized on the surface of PHBV/HA composite scaffolds.

Immobilization of collagen on PHBV/HA composite scaffolds was further confirmed by ESCA, as shown in Figure 3. The PHBV/HA composite scaffolds (Figure 3(b)) showed four peaks corresponding to C1s (binding energy, $285.0 \mathrm{eV}$ ), Ca2p (binding energy, $346.9 \mathrm{eV}$ ), P2p (binding energy, $102.4 \mathrm{eV}$ ), and $\mathrm{O} 1 \mathrm{~s}$ (binding energy, $531.7 \mathrm{eV}$ ). On the other hand, the PHBV/HA/Col composite scaffolds (Figure 3(c)) showed seven peaks corresponding to C1s, O1s, Ca2p, P2s, P2p (binding energy, P2s = 151.7 and $\mathrm{P} 2 \mathrm{p}=102.4 \mathrm{eV}$ ), N1s (binding energy, $400.0 \mathrm{eV}$ ), and Si2s (binding energy, $133.8 \mathrm{eV}$ ). The chemical composition of 


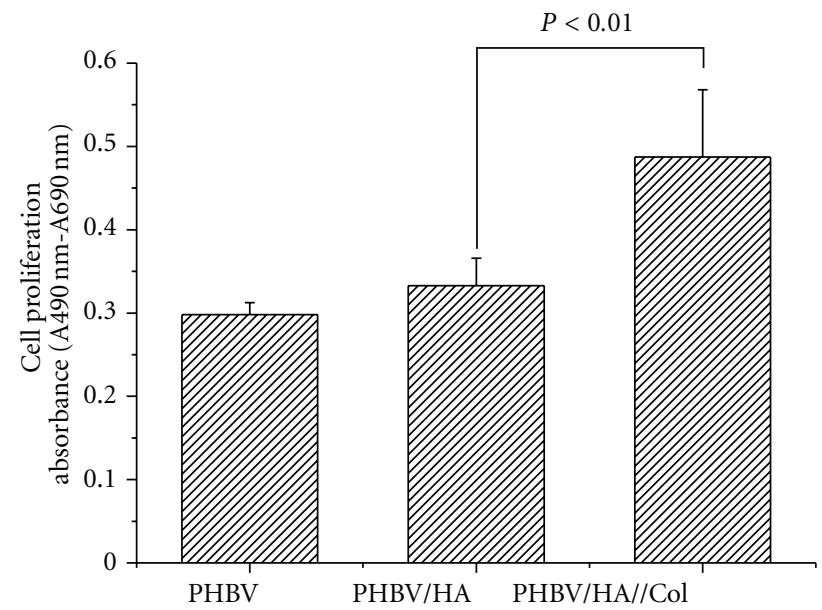

Figure 6: The proliferation of osteoblasts cultured on PHBV, PHBV/HA, and PHBV/HA/Col scaffolds for 3 days.

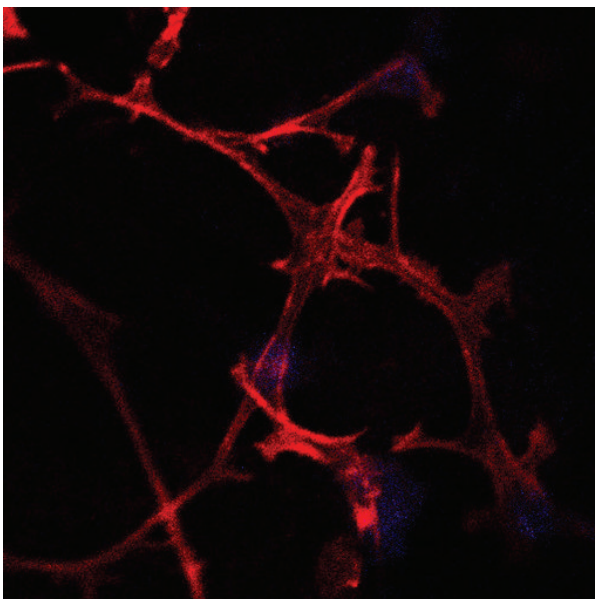

(a)

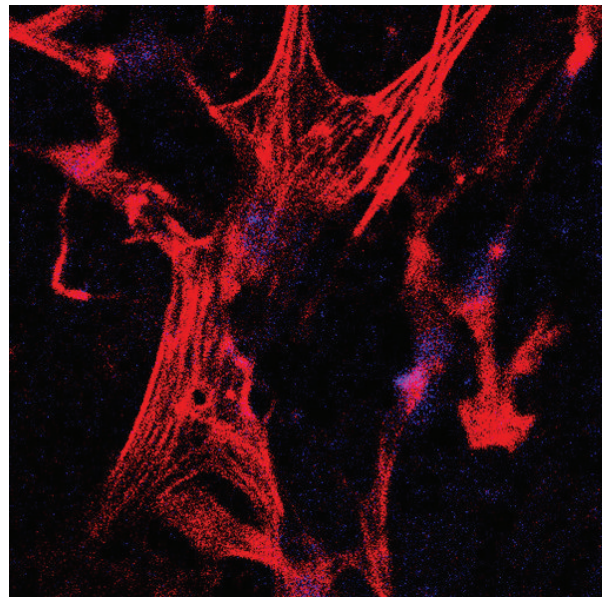

(b)

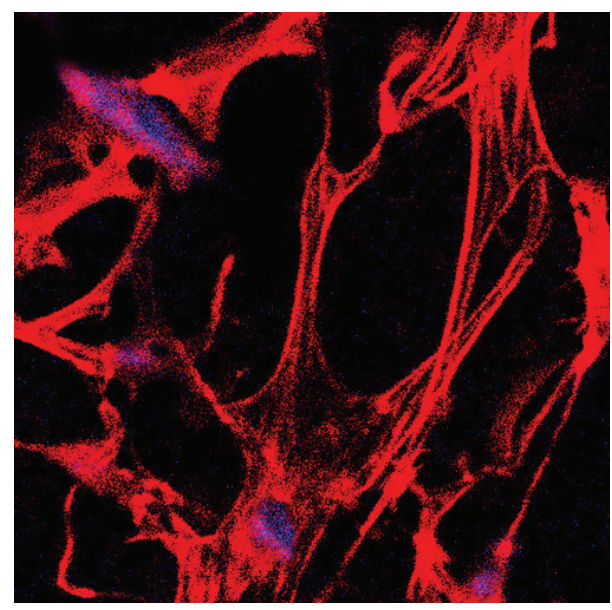

(c)

Figure 7: Confocal laser scanning micrographs (actin (red), nucleus (blue)) of osteoblast cells on PHBV (a), PHBV/HA (b), and PHBV/HA/Col (c) scaffolds after 3 days incubation (Mag: 400×). 


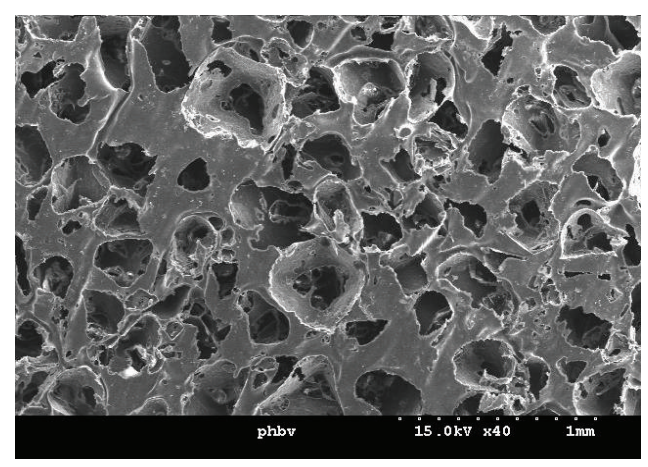

(a)

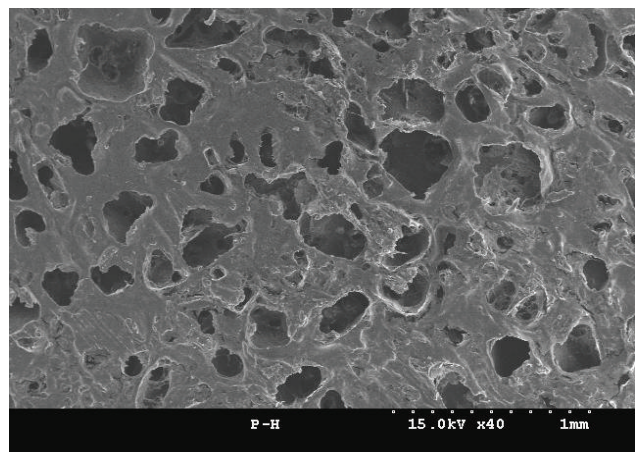

(c)

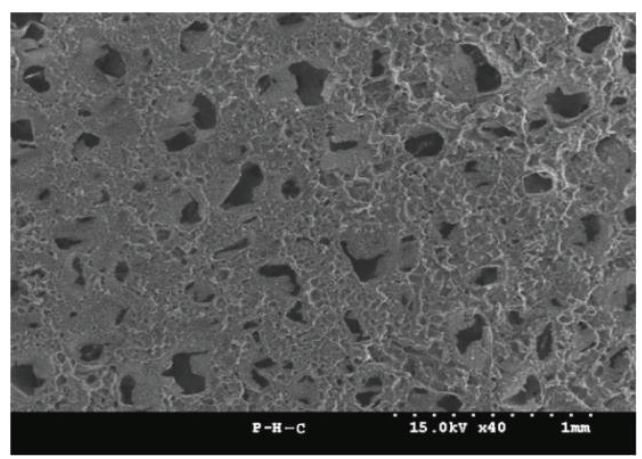

(e)

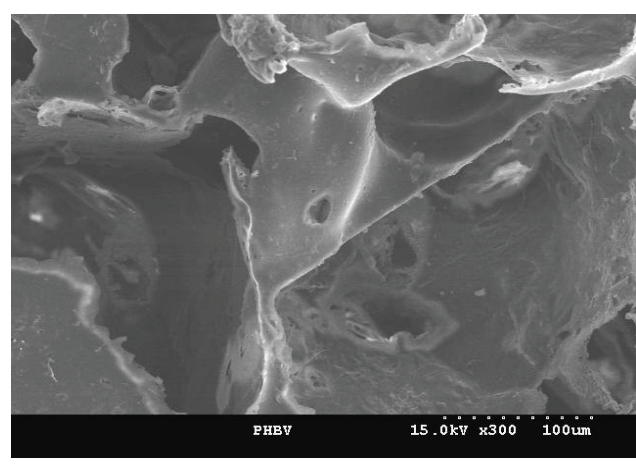

(b)

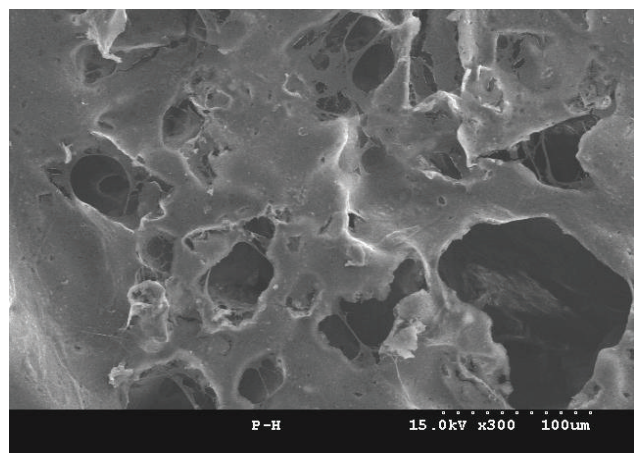

(d)

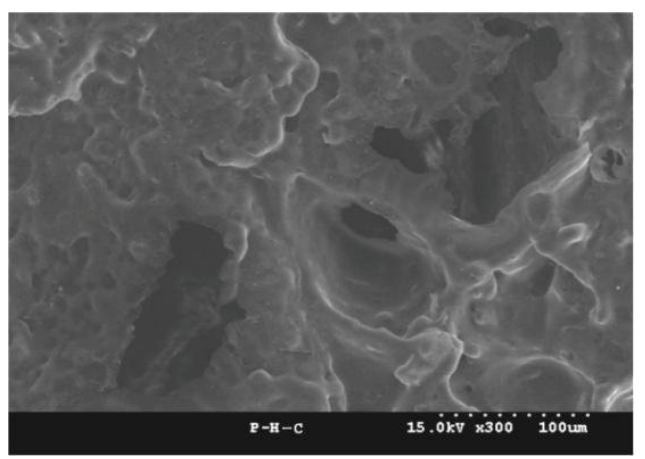

(f)

Figure 8: The SEM images of osteoblasts cultured on PHBV (a, b), PHBV/HA (c, d), and PHBV/HA/Col (e, f) scaffolds for 5 days (Mag 40× for (a), (c), (e); Mag 300× for (b), (d), (f)).

TABle 1: Atomic percent of the PHBV, PHBV-HA, and PHBV-HA-Col scaffolds.

\begin{tabular}{|c|c|c|c|c|c|c|c|c|}
\hline \multirow{2}{*}{ Substrate } & \multicolumn{8}{|c|}{ Atomic $\%$} \\
\hline & $\mathrm{C}$ & $\mathrm{O}$ & $\mathrm{Na}$ & $\mathrm{Cl}$ & $\mathrm{Ca}$ & $\mathrm{P}$ & $\mathrm{Si}$ & $\mathrm{N}$ \\
\hline PHBV & 68.1 & 31.7 & 0.1 & 0.1 & - & - & - & - \\
\hline PHBV/HA & 68.9 & 29.3 & 0.2 & 0.1 & 1.3 & 0.2 & - & - \\
\hline $\mathrm{PHBV} / \mathrm{HA} / \mathrm{Col}$ & 60.2 & 20.3 & 0.1 & 0.1 & 4.7 & 1.7 & 1.0 & 11.9 \\
\hline
\end{tabular}

the scaffolds calculated from the ESCA survey scan spectra is shown in Table 1. In the surface of the PHBV/HA/Col composite scaffolds (Figure 3(c)), two new elements such as nitrogen and silicon were observed, indicating the successful immobilization of collagen via a silanization with APTES.

The tensile properties of the porous PHBV and $\mathrm{PHBV} / \mathrm{HA}$ composites scaffolds are given in Figure 4. The modulus (0.95 MPa) and strength (1.8 MPa) of PHBV were significantly increased by the addition of HA nanosized particles up to 2.38 and $4.3 \mathrm{MPa}$, respectively, as shown in Figures 4(a) and 4(b). According to the report by Hull and clyne [33], the addition of HA nanoparticles had certainly reinforced the scaffolds as the incorporated HA is a stronger and stiffer material than PHBV. The values reported are 


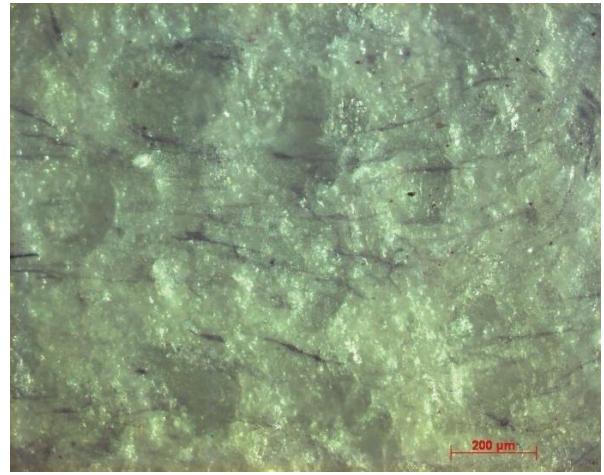

(a)

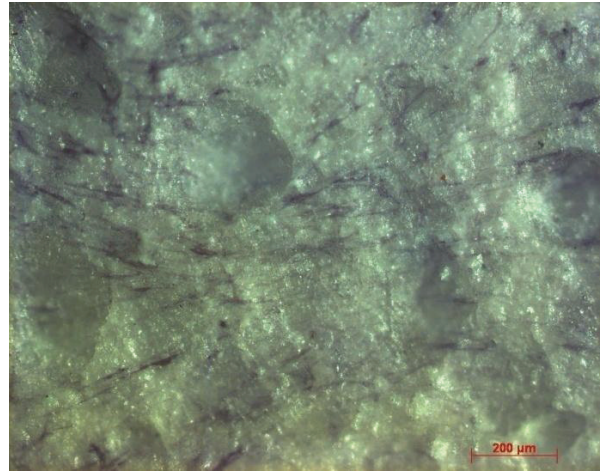

(b)

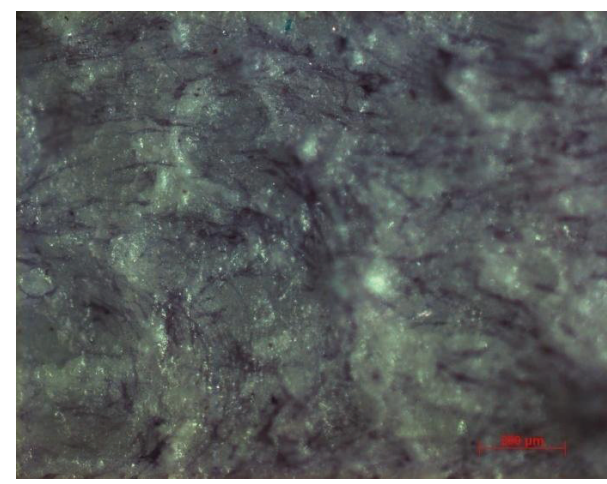

(c)

FIgure 9: ALP activity staining of osteoblasts (MCT3T-E1) cultured on PHBV (a), PHBV/HA (b), and PHBV/HA/Col (c) scaffolds for 16 days.

more similar to those reported for loadings of $20 \%$ of HA by weight $[9,34]$. Wang et al. produced the blends of $10 \%$ HA (micrometer sized) in a PHB solvent cast, salt particleleached scaffolds, and reported that the blend showed only small increases in modulus and stress [35]. In addition, it is possible that some nanoscale dispersion of the inorganic phase within the walls of the scaffold exists, and that these dispersed particles lead to the observed significant increased in the compressive modulus and strengths of the scaffold [10]. Overall, the incorporation of HA nanoparticles into the porous PHBV scaffolds enhances compressive mechanical properties of the scaffolds, which is beneficial for bone tissue engineering.

3.2. Bioactivity and Cellular Responses. The cell adhesion to the surface of $\mathrm{PHBV}, \mathrm{PHBV} / \mathrm{HA}$, and $\mathrm{PHBV} / \mathrm{HA} / \mathrm{Col}$ scaffolds was examined after 4 hours, 1 day, and 2 days incubation. Cells were stained with a cell-permeable green fluorescent dye (calcein AM) to observe cell adherence and cytoplasmic spreading. The morphologies of osteoblasts on the surface of the three scaffolds are shown in Figure 5. Few osteoblast cells adhered to the composite scaffolds after 4 hours of incubation (Figure 5(a), 5(b), 5(c)) and some cell spreading was also observed after 1 day (Figure 5(d), 5(e), $5(\mathrm{f})$ ) or 3 days (Figure 5(g), 5(h), 5(i)) of incubation. However, the osteoblasts adhered more quickly to the collagenimmobilized PHBV/HA scaffold than the PHBV/HA and
PHBV scaffolds. This result suggests that collagen immobilization improves cell adhesion to the surface of $\mathrm{PHBV} / \mathrm{HA}$ scaffolds. Figure 6 shows the proliferation of MC3T3-E1 cells cultured for 3 days on the composite scaffolds. The cell proliferation on the porous $\mathrm{PHBV} / \mathrm{HA} / \mathrm{Col}$ scaffolds was significantly $(P<0.05)$ higher than those on the PHBV and PHBV/HA scaffolds due to the immobilization of collagen. Figure 7 shows images of the cytoskeletal (actin) staining of osteoblasts cultured for 3 days on the PHBV (Figure 7(a)), PHBV/HA (Figure 7(b)), and PHBV/HA/Col (Figure 7(c)) composite scaffolds. Actin filaments are shown in red, and the nuclei are shown in blue. The cells cultured on the PHBV scaffold slightly expressed actin filaments. However, on the PHBV/HA scaffold, the cytoskeletons were organized (Figure $7(\mathrm{~b})$ ). On the $20 \mathrm{wt} \%$ HA scaffold, actin was more clearly organized with stress fibers (Figure $7(\mathrm{c})$ ). Figure 8 shows the SEM images of osteoblasts cultured on $\mathrm{PHBV}, \mathrm{PHBV} / \mathrm{HA}$, and PHBV/HA/Col scaffolds. After 5 days of culture, osteoblasts on the PHBV scaffolds were still isolated (Figure 8(a), 8(b)), whereas cells on the PHBV/HA scaffolds had formed a cytoplasmic web (Figure 8(c), 8(d)). However, the PHBV/HA/Col scaffolds were covered by an almost complete layer of osteoblasts with an almost flattened morphology (Figure 8(e), 8(f)). These results suggest that collagen chemically immobilized on PHBV scaffolds could provide a more conducive environment for cell growth. 
Differentiation of osteoblast cells is one of the key processes for bone regeneration. The in vitro differentiation of MC3T3-E1 into osteoblastic phenotype was qualitatively observed using ALP staining. In this study, ALP activity was determined as an indicator of osteoblastic differentiation of MC3T3-E1 cultured on PHBV, PHBV/HA, and PHBV/HA/Col composite scaffolds for 16 days after cell culturing. As shown in Figure 9, the amount of ALP synthesized by the cells cultured on the PHBV/HA/Col composite scaffolds was apparently higher (blue spot in figure) than those synthesized on the PHBV/HA and PHBV scaffolds. Since ALP is one of the most exclusive proteins synthesized by osteoblasts, the presence of ALP in MC3T3-E1 osteoblasts which were cultured on $\mathrm{PHBV} / \mathrm{HA} / \mathrm{Col}$ composite scaffolds could be used as the sole marker to confirm the osteoblastic phenotype of the cells [36].

\section{Conclusions}

The composite scaffolds composed of the porous PHBV and HA (PHBV/HA) were successfully fabricated by the slat-leaching technique. Collagen was immobilized on the surface of the porous PHBV/HA composite scaffolds (PHBV/HA/Col) for improving cell adhesion, and proliferation. It was found, from the experiments of cell proliferation, cytoskeletal organization, and alkaline phosphatase (ALP) activity, that the porous $\mathrm{PHBV} / \mathrm{HA} / \mathrm{Col}$ composite scaffolds showed a higher proliferation as well as a better production of ALP. This result suggests that the porous $\mathrm{PHBV} / \mathrm{HA} / \mathrm{Col}$ composite scaffolds have a high potential for use in the field of bone regeneration and tissue engineering.

\section{Acknowledgments}

This research was supported by the Basic Research Laboratory Program (2011) and by the Grant 2010-0011125 from the Ministry of Education, Science, and Technology of Korea.

\section{References}

[1] R. W. Lenz and R. H. Marchessault, "Bacterial polyesters: biosynthesis, biodegradable plastics and biotechnology," Biomacromolecules, vol. 6, no. 1, pp. 1-8, 2005.

[2] G. T. Köse, S. Ber, F. Korkusuz, and V. Hasirci, "Poly(3hydroxybutyric acid-co-3-hydroxyvaleric acid) based tissue engineering matrices," Journal of Materials Science, vol. 14, no. 2, pp. 121-126, 2003.

[3] G. Q. Chen and Q. Wu, "The application of polyhydroxyalkanoates as tissue engineering materials," Biomaterials, vol. 26, no. 33, pp. 6565-6578, 2005.

[4] Y. Tokiwa and B. P. Calabia, "Degradation of microbial polyesters," Biotechnology Letters, vol. 26, no. 15, pp. 11811189, 2004.

[5] M. Wang, Biomaterials and Tissue Engineering, Springer, Berlin, Germany, 2004, Edited by D. L. Shi.

[6] L. J. Chen and M. Wang, "Production and evaluation of biodegradable composites based on PHB-PHV copolymer," Biomaterials, vol. 23, no. 13, pp. 2631-2639, 2002.
[7] J. Ni and M. Wang, "In vitro evaluation of hydroxyapatite reinforced polyhydroxybutyrate composite," Materials Science and Engineering C, vol. 20, no. 1-2, pp. 101-109, 2002.

[8] Y. Liu and M. Wang, "Developing a composite material for bone tissue repair," Current Applied Physics, vol. 7, no. 5, pp. 547-554, 2007.

[9] N. Sultana and M. Wang, "Fabrication of HA/PHBV composite scaffolds through the emulsion freezing/freeze-drying process and characterisation of the scaffolds," Journal of Materials Science: Materials in Medicine, vol. 19, no. 7, pp. 2555-2561, 2008.

[10] K. S. Jack, S. Velayudhan, P. Luckman, M. Trau, L. Grøndahl, and J. Cooper-White, "The fabrication and characterization of biodegradable HA/PHBV nanoparticle-polymer composite scaffolds," Acta Biomaterialia, vol. 5, no. 7, pp. 2657-2667, 2009.

[11] S. Dasgupta, "Surface modification of polyolefins for hydrophilicity and bondability. Ozonization and grafting hydrophilic monomers on ozonized polyolefins," Journal of Applied Polymer Science, vol. 41, no. 1-2, pp. 233-248, 1990.

[12] Y. G. Ko, Y. H. Kim, K. D. Park et al., "Immobilization of poly(ethylene glycol) or its sulfonate onto polymer surfaces by ozone oxidation," Biomaterials, vol. 22, no. 15, pp. 2115-2123, 2001.

[13] J. O. Karlsson and P. Gatenholm, "Preparation and characterization of cellulose-supported HEMA hydrogels," Polymer, vol. 38, no. 18, pp. 4727-4731, 1997.

[14] J. C. Park, Y. S. Hwang, J. E. Lee et al., "Type I atelocollagen grafting onto ozone-treated polyurethane films: cell attachment, proliferation, and collagen synthesis," Journal of Biomedical Materials Research, vol. 52, no. 4, pp. 669-677, 2000.

[15] K. Anselme, "Osteoblast adhesion on biomaterials," Biomaterials, vol. 21, no. 7, pp. 667-681, 2000.

[16] J. Dobkowski, R. Kolos, J. Kaminski, and H. M. Kowalczynska, "Cell adhesion to polymeric surfaces: experimental study and simple theoretical approach," Journal of Biomedical Materials Research, vol. 47, no. 2, pp. 234-242, 1999.

[17] H. B. Lin, C. Garcia-Echeverria, S. Asakura, W. Sun, D. F. Mosher, and S. L. Cooper, "Endothelial cell adhesion on polyurethanes containing covalently attached RGD-peptides," Biomaterials, vol. 13, no. 13, pp. 905-914, 1992.

[18] A. Dekker, T. Beugeling, H. Wind et al., "Deposition of cellular fibronectin and desorption of human serum albumin during adhesion and spreading of human endothelial cells on polymers," Journal of Materials Science, vol. 2, no. 4, pp. 227233, 1991.

[19] J. P. Ranieri, R. Bellamkonda, J. Jacob, T. G. Vargo, J. A. Gardella, and P. Aebischer, "Selective neuronal cell attachment to a covalently patterned monoamine on fluorinated ethylene propylene films," Journal of Biomedical Materials Research, vol. 27, no. 7, pp. 917-925, 1993.

[20] C. Mauch, A. Hatamochi, K. Scharffetter, and T. Krieg, "Regulation of collagen synthesis in fibroblasts within a threedimensional collagen gel," Experimental Cell Research, vol. 178, no. 2, pp. 493-503, 1988.

[21] S. Nehrer, H. A. Breinan, A. Ramappa et al., "Chondrocyteseeded collagen matrices implanted in a chondral defect in a canine model," Biomaterials, vol. 19, no. 24, pp. 2313-2328, 1998.

[22] Y. Tesema, D. Raghavan, and J. Stubbs, "Bone cell viability on collagen immobilized poly(3-hydroxybutrate-co-3- hydroxyvalerate) membrane: Effect of surface chemistry," Journal of Applied Polymer Science, vol. 93, no. 5, pp. 2445-2453, 2004. 
[23] H. M. Kim, W. P. Chae, K. W. Chang et al., "Composite nanofiber mats consisting of hydroxyapatite and titania for biomedical applications," Journal of Biomedical Materials Research B, vol. 94, no. 2, pp. 380-387, 2010.

[24] M. Yamaura, R. L. Camilo, L. C. Sampaio, M. A. Macêdo, M. Nakamura, and H. E. Toma, "Preparation and characterization of (3-aminopropyl)triethoxysilane-coated magnetite nanoparticles," Journal of Magnetism and Magnetic Materials, vol. 279, no. 2-3, pp. 210-217, 2004.

[25] W. P. Chae, Z. C. Xing, Y. J. Kim, H. S. Sang, M. W. Huh, and I. K. Kang, "Fabrication and biocompatibility of rutincontaining PHBV nanofibrous scaffolds," Polymer, vol. 35, no. 3, pp. 210-215, 2011.

[26] K. Maghni, O. M. Nicolescu, and J. G. Martin, "Suitability of cell metabolic colorimetric assays for assessment of CD4+ $\mathrm{T}$ cell proliferation: comparison to 5-bromo-2-deoxyuridine (BrdU) ELISA," Journal of Immunological Methods, vol. 223, no. 2, pp. 185-194, 1999.

[27] Y. L. Cui, A. D. Qi, W. G. Liu et al., "Biomimetic surface modification of poly(L-lactic acid) with chitosan and its effects on articular chondrocytes in vitro," Biomaterials, vol. 24 , no. 21, pp. 3859-3868, 2003.

[28] M. J. Dalby, L. Di Silvio, E. J. Harper, and W. Bonfield, "Increasing hydroxyapatite incorporation into poly(methylmethacrylate) cement increases osteoblast adhesion and response," Biomaterials, vol. 23, no. 2, pp. 569-576, 2002.

[29] K. Isama and T. Tsuchiya, "Enhancing effect of poly(L-lactide) on the differentiation of mouse osteoblast-like MC3T3-E1 cells," Biomaterials, vol. 24, no. 19, pp. 3303-3309, 2003.

[30] Z. C. Xing, W. P. Chae, J. Y. Baek, M. J. Choi, Y. Jung, and I. K. Kang, "In vitro assessment of antibacterial activity and cytocompatibility of silver-containing phbv nanofibrous scaffolds for tissue engineering," Biomacromolecules, vol. 11, no. 5, pp. 1248-1253, 2010.

[31] W. Meng, S. Y. Kim, J. Yuan et al., "Electrospun PHBV/collagen composite nanofibrous scaffolds for tissue engineering," Journal of Biomaterials Science, vol. 18, no. 1, pp. 81-94, 2007.

[32] Y. Wang, Y. Ke, L. Ren, G. Wu, X. Chen, and Q. Zhao, "Surface engineering of PHBV by covalent collagen immobilization to improve cell compatibility," Journal of Biomedical Materials Research A, vol. 88, no. 3, pp. 616-627, 2009.

[33] D. Hull and T. W. Clyne, An Introduction to Composite Materials, Cambridge University Press, Cambridge, UK, 2nd edition, 1996.

[34] S. Coskun, F. Korkusuz, and V. Hasirci, "Hydroxyapatite reinforced poly(3-hydroxybutyrate) and poly(3-hydroxybutyrateco-3-hydroxyvalerate) based degradable composite bone plate," Journal of Biomaterials Science, vol. 16, no. 12, pp. 14851502, 2005.

[35] Y. W. Wang, Q. Wu, J. Chen, and G. Q. Chen, "Evaluation of three-dimensional scaffolds made of blends of hydroxyapatite and poly(3-hydroxybutyrate-co-3-hydroxyhexanoate) for bone reconstruction," Biomaterials, vol. 26, no. 8, pp. 899 904, 2005.

[36] H. Fong and D. H. Reneker, "Electrospinning and the formation of nanofibers," in Structure Formation in Polymeric Fibers, D. R. Salem, Ed., pp. 225-246, Hanser Gardner, Munich, Germany, 2001. 

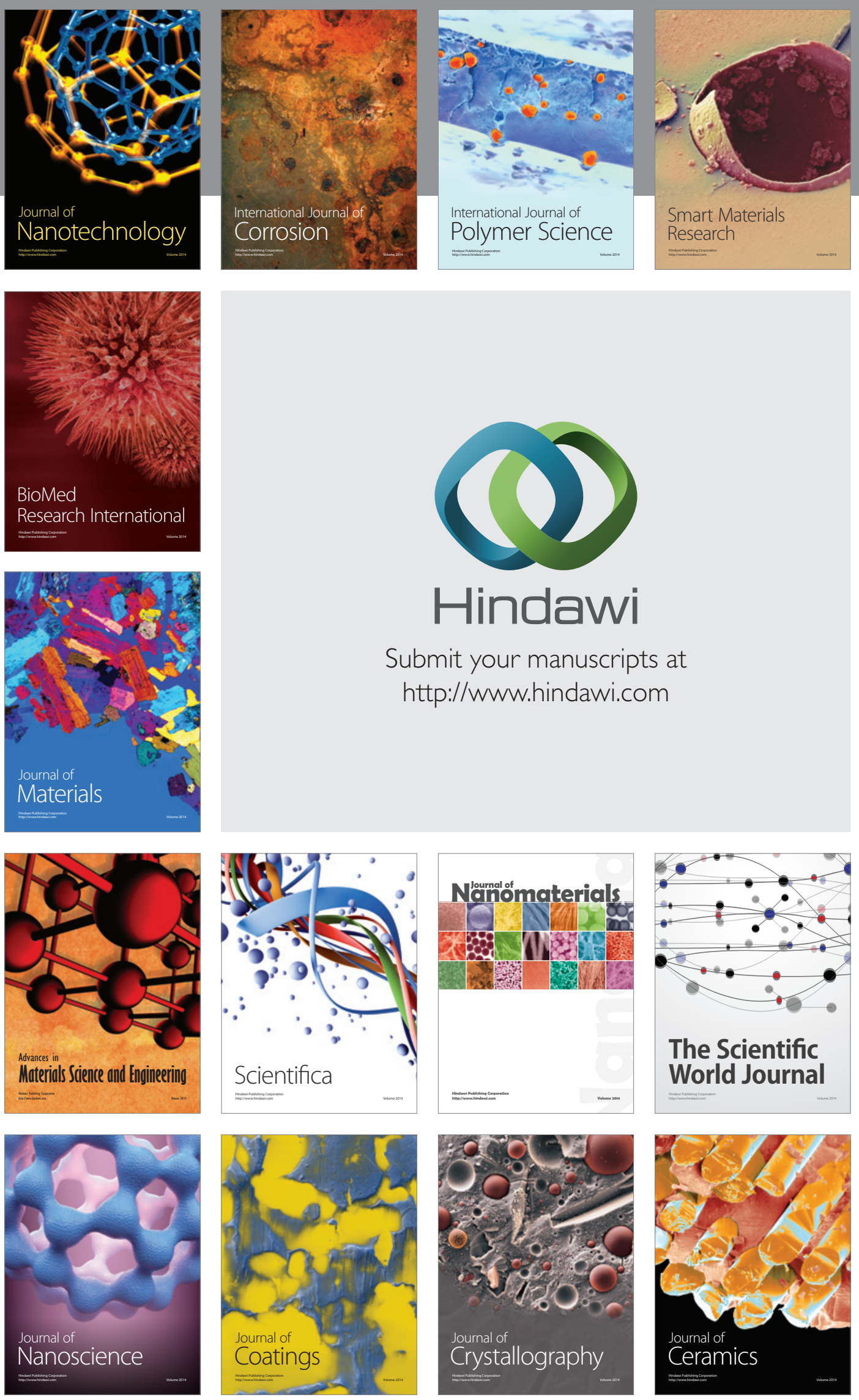

The Scientific World Journal

Submit your manuscripts at

http://www.hindawi.com

\section{World Journal}

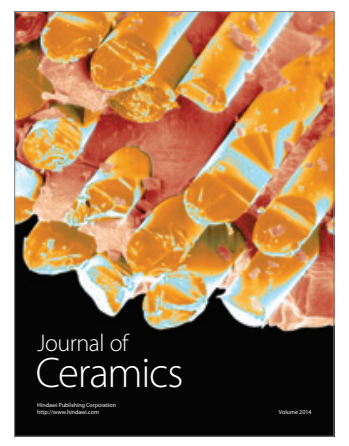

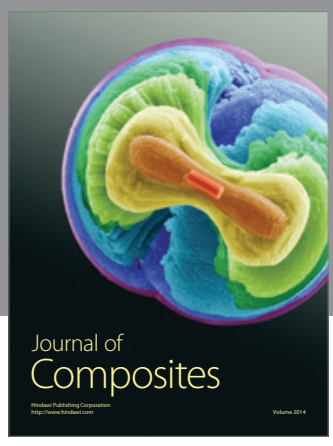
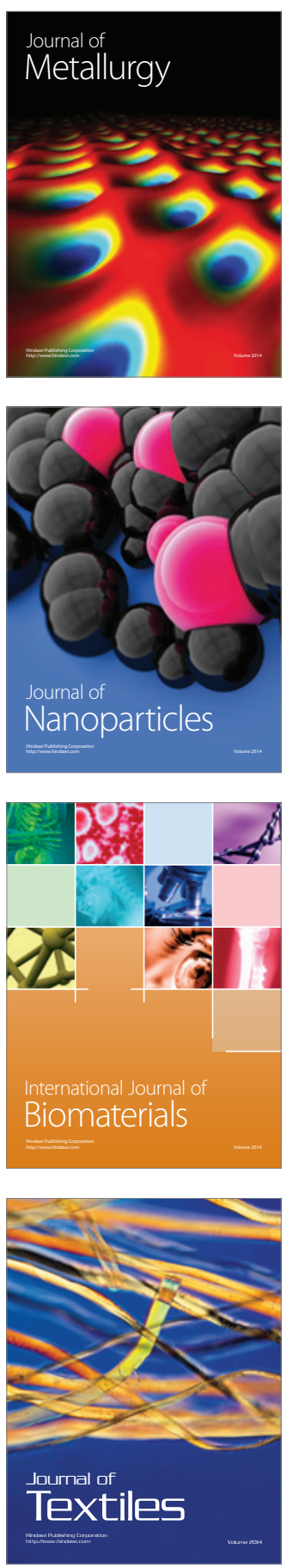